\title{
Finite $N_{\mathrm{c}}$ corrections in the Balitsky-Kovchegov equation at next-to-leading order
}

\section{T. Lappi, H. Mäntysaari and A. Ramnath*}

Department of Physics, University of Jyväskylä P.O. Box 35, 40014 University of Jyväskylä, Finland Helsinki Institute of Physics, P.O. Box 64, 00014 University of Helsinki, Finland

E-mail: tuomas.v.v.lappi@jyu.fi, heikki.mantysaari@jyu.fi, anramnat@student.jyu. fi

We study the finite- $N_{\mathrm{c}}$ corrections to the next-to-leading order (NLO) Balitsky-Kovchegov (BK) equation. This contains correlators of six Wilson lines, which we express in terms of the two-point function using the Gaussian approximation. Numerically, the effects of these finite- $N_{\mathrm{c}}$ corrections on the NLO BK equation are found to be smaller than the expected $1 / N_{\mathrm{c}}^{2} \sim 10 \%$. Corrections may be large for individual correlators, but have less of an influence on the shape of the amplitude as a function of the dipole size. There is an even smaller effect on the evolution speed as a function of rapidity.

HardProbes 2020

1-6 June 2020

Austin, Texas

${ }^{*}$ Speaker 


\section{Introduction}

The Color Glass Condensate effective field theory is used to describe QCD in the region where parton densities grow to the order of the inverse of the QCD coupling. Cross sections for scattering processes can be expressed in this framework in terms of $n$-point correlators of Wilson lines these describe the eikonal propagation of a parton in the target field. The energy dependence of the target is obtained by solving the Balitsky-Kovchegov (BK) equation for the 2-point correlator, which was derived to next-to-leading order (NLO) accuracy in Ref. [1]. The NLO BK equation is known to be unstable [2] and that its finite- $N_{\mathrm{c}}$ corrections are smaller than the naive expectation of $O\left(1 / N_{\mathrm{c}}^{2}\right)$ [3, 4]. It contains six-point correlators, for which we derive analytical parametric equations using the Gaussian approximation [5-7]. We study numerically the effect of finite- $N_{\mathrm{c}}$ corrections from the six-point correlators on NLO BK evolution. For the full details, see [8].

\section{NLO BK correlators using the Gaussian approximation}

For any product of $n / 2$ pairs of fundamental Wilson lines $U U^{\dagger}$, we use the notation $S_{\boldsymbol{x}_{1}, \boldsymbol{x}_{2}, \ldots, \boldsymbol{x}_{n-1}, \boldsymbol{x}_{n}}^{(n)}:=\operatorname{tr}\left(U_{\boldsymbol{x}_{1}} U_{\boldsymbol{x}_{2}}^{\dagger} \ldots U_{\boldsymbol{x}_{\mathrm{n}-1}} U_{\boldsymbol{x}_{n}}^{\dagger}\right) / N_{\mathrm{c}}$. The NLO BK equation (with $n_{f}=0$ ) reads [1]

$$
\partial_{Y}\left\langle S_{\boldsymbol{x}, \boldsymbol{y}}^{(2)}\right\rangle=\frac{\alpha_{\mathrm{s}} N_{\mathrm{c}}}{2 \pi^{2}} \int_{\boldsymbol{z}} K_{1}^{\mathrm{BC}}\left\langle D_{1}\right\rangle+\frac{\alpha_{\mathrm{s}}^{2} N_{\mathrm{c}}^{2}}{16 \pi^{4}} \int_{z, \boldsymbol{z}^{\prime}}\left(K_{2,1}\left\langle D_{2,1}\right\rangle+K_{2,2}\left\langle D_{2,2}\right\rangle\right)+O\left(n_{f}\right),
$$

where the brackets \langle\rangle refer to the expectation value over target color field configurations. The kernels are simple functions of $r^{2}=(\boldsymbol{x}-\boldsymbol{y})^{2}, X^{2}=(\boldsymbol{x}-\boldsymbol{z})^{2}, X^{\prime 2}=\left(\boldsymbol{x}-\boldsymbol{z}^{\prime}\right)^{2}, Y^{2}=(\boldsymbol{y}-\boldsymbol{z})^{2}, Y^{\prime 2}=\left(\boldsymbol{y}-\boldsymbol{z}^{\prime}\right)^{2}$ and $Z^{2}=\left(z-z^{\prime}\right)^{2}$. The Wilson line operators are

$$
\begin{aligned}
\left\langle D_{1}\right\rangle=\left\langle S_{\boldsymbol{x}, \boldsymbol{z}}^{(2)} S_{\boldsymbol{z}, \boldsymbol{y}}^{(2)}\right\rangle-\left\langle S_{\boldsymbol{x}, \boldsymbol{y}}^{(2)}\right\rangle, \quad\left\langle D_{2,1}\right\rangle & =\left\langle S_{\boldsymbol{x}, \boldsymbol{z}}^{(2)} S_{\boldsymbol{z}, \boldsymbol{z}^{\prime}}^{(2)} S_{\boldsymbol{z}^{\prime} \boldsymbol{y}}^{(2)}\right\rangle-\frac{1}{N_{\mathrm{c}}^{2}}\left\langle S_{\boldsymbol{x}, \boldsymbol{z}, \boldsymbol{z}^{\prime}, \boldsymbol{y}, \boldsymbol{z}, \boldsymbol{z}^{\prime}}^{(6)}\right\rangle-\left(\boldsymbol{z}^{\prime} \rightarrow \boldsymbol{z}\right), \\
\left\langle D_{2,2}\right\rangle & =\left\langle S_{\boldsymbol{x}, \boldsymbol{z}}^{(2)} S_{\boldsymbol{z}, \boldsymbol{z}^{\prime}}^{(2)} S_{\boldsymbol{z}^{\prime}, \boldsymbol{y}}^{(2)}\right\rangle-\left(\boldsymbol{z}^{\prime} \rightarrow \boldsymbol{z}\right) .
\end{aligned}
$$

We refer to two pieces of the right side of Eq. (1) as the "LO-like" contribution $\frac{\alpha_{\mathrm{s}} N_{\mathrm{c}}}{2 \pi^{2}} K_{1}^{\mathrm{BC}} \otimes\left\langle D_{1}\right\rangle$ and the "NLO-like" contribution $\frac{\alpha_{\mathrm{s}}^{2} N_{\mathrm{c}}^{2}}{16 \pi^{4}} K_{2,1} \otimes\left\langle D_{2,1}\right\rangle+\frac{\alpha_{\mathrm{s}}^{2} N_{\mathrm{c}}^{2}}{16 \pi^{4}} K_{2,2} \otimes\left\langle D_{2,2}\right\rangle$. In the large- $N_{\mathrm{c}}$ limit, one can drop terms suppressed by $1 / N_{\mathrm{c}}$. Then averages of products factorize into products of averages.

In the Gaussian approximation, all correlators are parametrized by a two-point function as

$$
\langle O[U]\rangle_{\eta}=\exp \left\{-\frac{1}{2} \int^{\eta} \mathrm{d} \tilde{\eta} \int_{\boldsymbol{u}_{1}, \boldsymbol{u}_{2}} G_{\boldsymbol{u}_{1}, \boldsymbol{u}_{2}}(\tilde{\eta}) L_{\boldsymbol{u}_{1}}^{a} L_{\boldsymbol{u}_{2}}^{a}\right\} O[U]
$$

where $\int_{\boldsymbol{u}}=\int \mathrm{d}^{2} \boldsymbol{u}, \eta$ is a parametrization rapidity and $L_{\boldsymbol{u}}^{a}$ is a Lie derivative that acts on Wilson lines according to $L_{\boldsymbol{u}}^{a} U_{\boldsymbol{x}}=-i g \delta^{(2)}(\boldsymbol{x}-\boldsymbol{u}) t^{a} U_{\boldsymbol{x}}$. In the case of $n$-point correlators larger than the dipole, $\langle O[U]\rangle$ is an $n \times n$ matrix of correlators, denoted $\mathcal{A}(\eta)$ and the differential form of Eq. (4) becomes an $n \times n$ matrix differential equation $\partial_{\eta} \mathcal{A}(\eta)=-\mathcal{M}(\eta) \mathcal{A}(\eta)$.

The procedure to find parametric equations for the six-point correlators is as follows. First, we choose a multiplet basis $\boldsymbol{B}$ for the space of all six-point correlators. Then, we construct the correlator matrix $\mathcal{A}$ by taking $\boldsymbol{B}\left(U_{\boldsymbol{z}} \otimes U_{\boldsymbol{z}^{\prime}}^{\dagger} \otimes U_{\boldsymbol{v}} \otimes U_{\boldsymbol{y}}^{\dagger} \otimes U_{\boldsymbol{x}} \otimes U_{\boldsymbol{w}}^{\dagger}\right) \boldsymbol{B}^{\mathrm{T}}$. Next, we construct the transition matrix $\mathcal{M}$ by summing (for each element in $\mathcal{A}$ ) all possible one-gluon diagrams obtained with the double Lie derivative operator and rewriting the result in terms of elements of $\mathcal{A}$. 
The most efficient way to construct a basis is to exploit the structure of the six-point correlators that are actually needed for the NLO BK equation, which only have four distinct coordinates. It is then easy to see that there is one way to join the endpoints of six Wilson lines with these coordinates such that four Wilson lines cancel (due to unitarity) and only a dipole remains. Similarly, the choice of two more particular basis elements results in two correlators that reduce to four-point correlators. The remaining three basis elements are chosen such that they are orthonormal to the first three.

The differential equation, which has an exponential solution, now decouples into three independent equations. Instead of exponentiating a six-by-six matrix; at most we will need to exponentiate a three-by-three matrix, which can be done analytically. The first transition sub-matrix gives the parametrization for the dipole correlator. The second transition sub-matrix gives the parametrization for the four-point correlator with one repeated coordinate [6]. Taking linear combinations of the elements of the last transition sub-matrix gives the six-point correlators required.

To verify the validity of our parametric equations, we perform two checks. Firstly, the Gaussian approximation has the built-in property that it should be consistent in color algebra. Secondly, when the equation for $\left\langle S^{(2)} S^{(2)} S^{(2)}\right\rangle$ is taken in the dilute limit, it should be the same up to second order as the parametric equation for the large- $N_{\mathrm{c}}$ counterpart operator.

\section{Numerical results}

In Fig. 1, we plot $\left\langle D_{2,1}\right\rangle$ and $\left\langle D_{2,2}\right\rangle$. Since both quantities reduce to the same expression in the large- $N_{\mathrm{c}}$ limit, only one curve is shown for the large- $N_{\mathrm{c}}$ case. The dashed curves show the differences between large and finite $N_{\mathrm{c}}$ for each quantity. The finite- $N_{\mathrm{c}}$ corrections are negligible at $a Q_{s} \ll 1$, but become numerically important when $a Q_{s} \gtrsim 1$. At $a Q_{s}=1$, the finite- $N_{\mathrm{c}}$ corrections to $\left\langle D_{2,1}\right\rangle$ and $\left\langle D_{2,2}\right\rangle$ are approximately $20 \%$ and $16 \%$, respectively. In comparison, the LO-like operator $\left\langle D_{1}\right\rangle$ has a finite- $N_{\mathrm{c}}$ correction of approximately $8 \%$. Since the evolution is driven by the dipole sizes $r \lesssim 1 / Q_{s}$, the effect of the $1 / N_{\mathrm{c}}^{2}$ suppressed contributions to the evolution can be smaller.

In Fig. 2, we plot the difference between the large- $N_{\mathrm{c}}$ and finite- $N_{\mathrm{c}}$ cases, separately for the LO-like and NLO-like terms. The difference has the opposite sign in the LO-like and the NLO-like terms and so part of the difference cancels in the total evolution speed. At $r Q_{s}=1$, the relative finite- $N_{\mathrm{c}}$ correction is approximately $8 \%$ in the LO-like contribution and $13 \%$ in the NLO-like contribution. The relative magnitude of the total $1 / N_{\mathrm{c}}^{2}$ suppressed contribution is $5 \%$.

In Fig. 3, we show the ratio of $N$ obtained by solving the full NLO BK equation at finite $N_{\mathrm{c}}$ to that at large $N_{\mathrm{c}}$. At $r \gtrsim 1 / Q_{s}$, when the details of the initial condition are lost and one enters the geometric scaling region, the difference between the large- $N_{\mathrm{c}}$ and finite- $N_{\mathrm{c}}$ cases evolves only very slowly. At small dipoles, the ratio grows approximately linearly in $Y$. The fact that the total finite- $N_{\mathrm{c}}$ correction is positive at small dipole sizes and negative at large dipoles, as seen in Fig. 2, is found to hold also asymptotically after many units of rapidity evolution.

The evolution speed of the saturation scale, $\partial_{Y} \ln Q_{s}^{2}$, is shown in Fig. 4. The finite- $N_{\mathrm{c}}$ corrections are seen to be more important at the initial condition, slowing down the evolution of $Q_{s}^{2}$ by approximately $5 \%$. Later in the evolution, the difference becomes smaller - of the order of $1 \%$. 


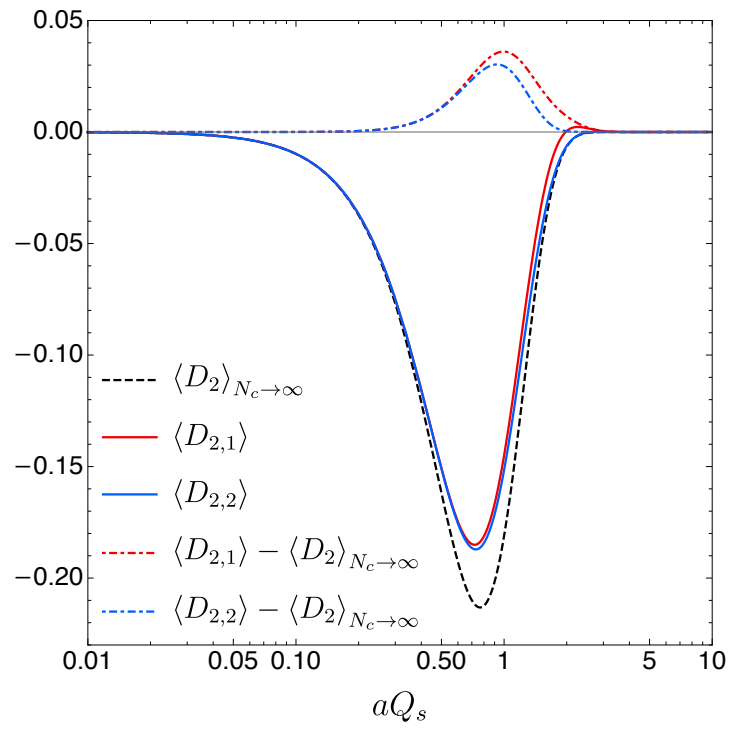

Figure 1: Correlator factors $\left\langle D_{2,1}\right\rangle$ and $\left\langle D_{2,2}\right\rangle$ in the NLO-like part of the BK equation (1), in the line configuration of coordinates. Both factors reduce to the same expression $\left\langle D_{2}\right\rangle_{\text {Large }} N_{\mathrm{c}}$ in the large- $N_{\mathrm{c}}$ limit, as also shown in the figure.

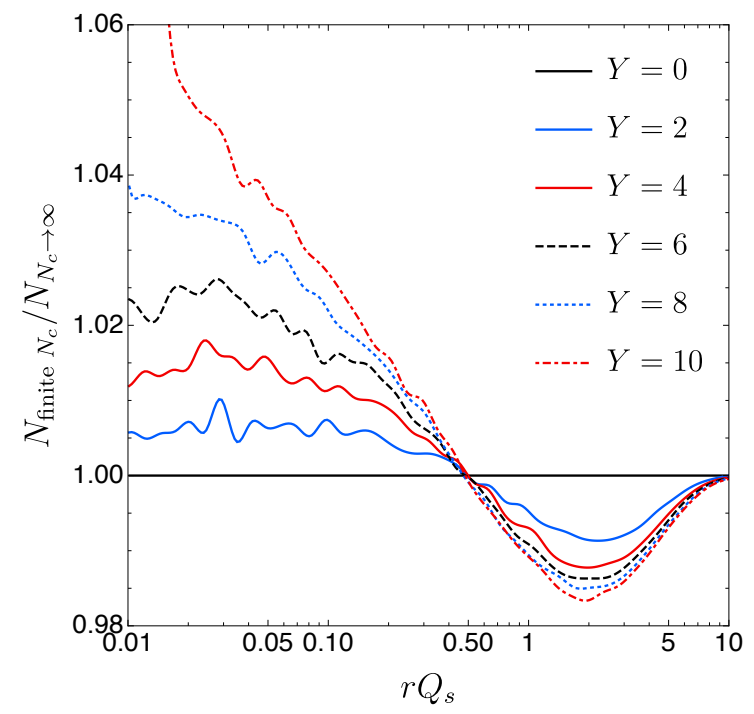

Figure 3: Evolution for the ratio of the dipole amplitudes obtained by performing the finite- $N_{\mathrm{c}}$ and large$N_{\mathrm{c}}$ evolutions with the same initial condition.

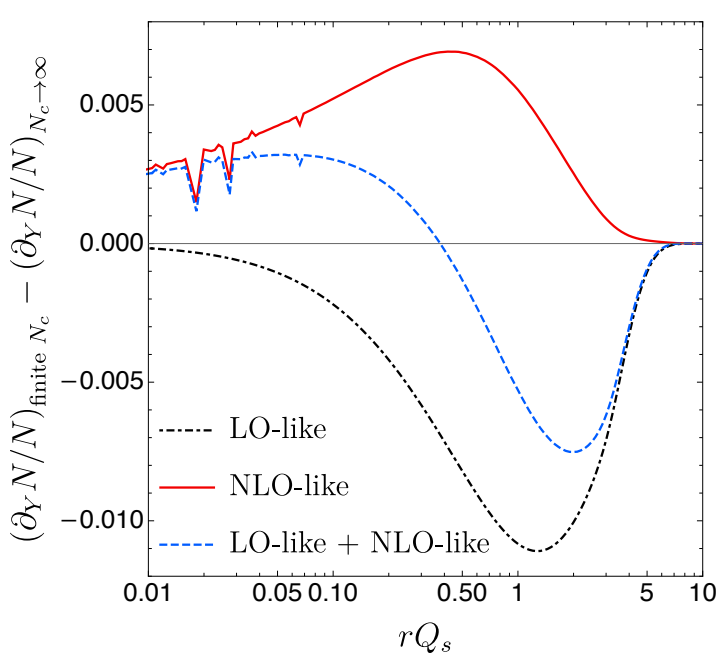

Figure 2: Difference of the evolution speeds at finite $N_{\mathrm{c}}$ and at large $N_{\mathrm{c}}$, shown separately for the LO-like, NLO-like and total (LO-like + NLO-like) contributions.

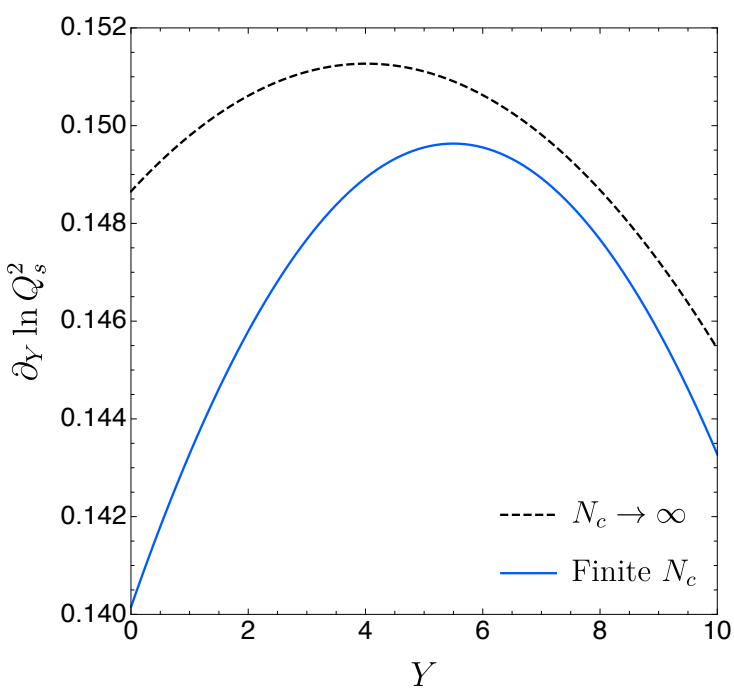

Figure 4: Evolution speed of the saturation scale $Q_{s}^{2}$ as a function of rapidity at large $N_{\mathrm{c}}$ and at finite $N_{\mathrm{c}}$.

\section{Conclusions}

The overall finite- $N_{\mathrm{c}}$ corrections to the NLO-like part of the BK equation are somewhat smaller than what is naively expected. However, they need to be considered carefully in the NLO BK equation, since they may have a non-negligible effect at the required accuracy. When 
correlators are considered between Wilson lines separated by large distances relative to $1 / Q_{\mathrm{s}}$, $1 / N_{\mathrm{c}}^{2}$ suppressed corrections may be considerable. Despite these potentially large corrections to individual correlators, these configurations do not contribute much to the right side of the BK equation. There is a small but significant effect on the shape of the dipole amplitude as a function of $r$. The finite- $N_{\mathrm{c}}$ corrections are watered down further when one considers the evolution speed of $Q_{\text {s }}$ as a function of rapidity.

\section{Acknowledgements}

This work was supported by the Academy of Finland, projects 314764 (H. M.) and 321840 (T. L.), and by the National Research Foundation of South Africa (A. R.). A. R. and T. L. are supported by the European Research Council (ERC) under the European Unions Horizon 2020 research and innovation programme (grant agreement No. ERC-2015-CoG-681707). The content of this article does not reflect the official opinion of the European Union and responsibility for the information and views expressed therein lies entirely with the authors. Computing resources from CSC - IT Center for Science in Espoo, Finland and from the Finnish Grid and Cloud Infrastructure (persistent identifier urn:nbn: $f i$ :research-infras-2016072533) were used in this work.

\section{References}

[1] I. Balitsky and G. A. Chirilli, Next-to-leading order evolution of color dipoles, Phys. Rev. D77 (2008) 014019 [arXiv:0710.4330 [hep-ph]].

[2] T. Lappi and H. Mäntysaari, Direct numerical solution of the coordinate space Balitsky-Kovchegov equation at next to leading order, Phys. Rev. D91 (2015) no. 7074016 [arXiv: 1502.02400 [hep-ph]].

[3] K. Rummukainen and H. Weigert, Universal features of JIMWLK and BK evolution at small X, Nucl. Phys. A739 (2004) 183 [arXiv:hep-ph/0309306 [hep-ph]].

[4] Y. V. Kovchegov, J. Kuokkanen, K. Rummukainen and H. Weigert, Subleading-N(c) corrections in non-linear small-x evolution, Nucl. Phys. A823 (2009) 47 [arXiv: 0812 . 3238 [hep-ph]].

[5] H. Fujii, F. Gelis and R. Venugopalan, Quark pair production in high energy pA collisions: General features, Nucl. Phys. A780 (2006) 146 [arXiv: hep-ph/0603099 [hep-ph]].

[6] C. Marquet and H. Weigert, New observables to test the Color Glass Condensate beyond the large- $N_{c}$ limit, Nucl. Phys. A843 (2010) 68 [arXiv: 1003.0813 [hep-ph]].

[7] F. Dominguez, C. Marquet, B.-W. Xiao and F. Yuan, Universality of Unintegrated Gluon Distributions at small x, Phys. Rev. D83 (2011) 105005 [arXiv: 1101.0715 [hep-ph]].

[8] T. Lappi, H. Mäntysaari and A. Ramnath, Next-to-leading order balitsky-kovchegov equation beyond large $n_{\mathrm{c}}$, arXiv:2007.00751 [hep-ph]. 